\title{
Spontaneous Resolution of Vitreomacular Traction in Two Patients with Diabetic Macular Edema
}

\author{
David R.P. Almeida Eric K. Chin Stephen R. Russell
}

Vitreoretinal Service, Department of Ophthalmology and Visual Sciences, University of Iowa Hospitals and Clinics, Iowa City, Iowa, USA

\section{Key Words}

Vitreomacular adhesion - Vitreomacular traction · Diabetic macular edema

\section{Abstract}

The nature of the vitreoretinal interface in conditions like diabetic macular edema (DME) remains incompletely understood. Furthermore, it is not clear what the role of pharmacological enzymatic vitreolysis will play in cases of vitreomacular traction (VMT) associated with macular disease like DME. We describe the spontaneous resolution of VMT in 2 patients with DME. As both surgical and pharmacologic interventions have been suggested to treat DME in the setting of VMT, we feel that a clarification of the nomenclature and reporting of these cases of natural history may be useful in more fully understanding the complex decisionmaking involved when determining whether to treat this subset of patients.

(C) 2014 S. Karger AG, Basel

\section{Introduction}

Vitreomacular traction (VMT) or VMT syndrome is an increasingly recognized disorder that has attracted recent attention as a target for both surgical and pharmacologic treatments [1]. Despite recent advances, however, the nature and character of the vitreoretinal junction remains incompletely understood [2]. Furthermore, the nomenclature used to describe disorders of this interface may be confusing and subject to imprecise characterization. VMT may be found with increased incidence in disorders that involve disordered vitreoretinal adhesion such as diabetic retinopathy or foreshortening of the vitreous as

David R.P. Almeida, MD, MBA, PhD, FRCSC

Department of Ophthalmology and Visual Sciences

University of Iowa Hospitals and Clinics

200 Hawkins Drive, PFP Room 11196-D, Iowa City, IA 52240 (USA)

E-Mail dalmeida@evolation-medical.com 
Almeida et al.: Spontaneous Resolution of Vitreomacular Traction in Two Patients with Diabetic Macular Edema

partial vitreous detachment [3]. Isolated VMT with decreased vision loss - termed VMT syndrome - occurs in isolation without associated macular disease; however, it is recognized that symptomatic vitreomacular adhesion (sVMA) may be associated with other conditions such as macular hole (MH), epiretinal membrane (ERM), age-related macular degeneration (AMD), diabetic macular edema (DME), or retinal vein occlusion (RVO) [3-5].

Both VMT and its pathologic cousin sVMA require evidence of vitreomacular attachment and vitreous tension; moreover, in the case of sVMA, vision loss typically manifested as blurring, reduced acuity, or metamorphopsia is present [1]. VMT may be confirmed only when the vitreous tensional force is sufficient to result in characteristic retinal or vitreous structural changes, which is best identified on optical coherence tomography (OCT). Such changes include widespread to point-like macular retinal thickening (including an increase in intraretinal fluid), tenting of the inner limiting membrane and/or other inner retinal tissues, and/or focal tractional macular detachment(s) $[3,6]$. Symptoms of retinal distortion in the absence of VMT are usually due to other disorders, most commonly ERMs or subretinal or intraretinal fluid.

Recent attempts to define photographic standards relating to the OCT abnormalities associated with VMA that may result in VMT has been proposed by Simpson et al. [5]. We have found that for purposes of defining inclusion criteria for studies and general thresholds of disease definition, these standards may be useful, although they represent a starting point in our quantification of image-disorder correlation.

Intravitreal ocriplasmin (Jetrea ${ }^{\circledR}$ ) was approved in the USA in October 2012 to relieve VMA causing VMT and MH, and just recently, in August 2013, has been approved in Canada based on several clinical trials [7-10]. Ocriplasmin is primarily used for the treatment of stage 2 MHs less than $400 \mu \mathrm{m}$. Nonetheless, it is not clear what the role of pharmacological enzymatic vitreolysis will play in cases of VMA associated with macular disease like DME.

In this report, we describe the spontaneous resolution of VMT in two eyes of 2 patients with DME. As both surgical and pharmacologic intervention have been suggested to treat DME in the setting of VMT, we feel that a clarification of the nomenclature and reporting of these cases of natural history may be useful in more fully understanding the complex decision-making involved when determining whether to treat this subset of patients.

This study was conducted in full adherence to the Declaration of Helsinki and all federal and state laws.

\section{Case Presentation}

Case 1 is a 63-year-old white male, previously treated for proliferative diabetic retinopathy with panretinal photocoagulation. Additionally, the patient had persistent DME OU with stable vision after focal laser photocoagulation and anti-vascular endothelial growth factor. His visual acuity in OS was 6/9, and he had macular thickening diagnosed as mild diffuse DME [central macular thickness (CMT) $344 \mu \mathrm{m}$ ]. The OCT also demonstrated inner retinal tenting, confirming VMT OS (fig. 1). Because his edema and visual acuity were stable, observation was recommended. Six months later, the patient returned and had developed spontaneous resolution of VMT and an improved visual acuity of 6/6 OS with no interval interventions. Of note, the macula remained thickened (CMT $325 \mu \mathrm{m}$ ) and cystic (fig. 1) OS.

Case 2 is a 68-year-old white male with non-proliferative diabetic retinopathy OU and DME OD. In OD, he had a visual acuity of 6/12, mild DME (CMT $314 \mu \mathrm{m}$ ), and OCT evidence of VMT (fig. 2). Similar to Case 1, the acuity and retinal thickness were stable and observation was recommended. Six months later, VMT had spontaneously resolved with no interval 
Almeida et al:: Spontaneous Resolution of Vitreomacular Traction in Two Patients with Diabetic Macular Edema

treatments during the follow-up period. The visual acuity improved to 6/7.5 in OD, but the mild cystic DME was unchanged (CMT $314 \mu \mathrm{m}$ ) on OCT.

\section{Discussion}

Studies indicate that prolonged VMT may lead to progressive vision loss [6]; however, while treatment decision-making for MH and ERM associated with VMT is more straightforward based on current evidence, in cases of VMT in the context of DME, AMD, and RVO, natural history is poorly understood and significant gaps in the literature exist. We demonstrate 2 cases with a history of DME and VMT who had spontaneous resolution of VMT with concomitant improvement in their visual acuity with no associated intervention over the course of 6 months. It necessitates emphasizing that in both cases, visual acuity and VMT were inversely correlated; however, cystic retinal changes in DME may be consistent with $6 / 6$ vision. Upon resolution of VMT, both visual acuity and symptomatology improved despite abnormalities in macular morphology. This evidence supports VMT as a contributor to the underlying pathology limiting visual acuity. Improvement in visual acuity can thus be postulated to be partly derived from the release of VMT.

VMA may contribute to the pathogenesis of several macular diseases and it has been recently estimated that the total number of eye diseases that are caused by, or associated with, VMA ranges from 0.35 to $1.5 \%$ of the population [5]. This range, even at the lower end, is an astoundingly immense number, which may be difficult to triage, treat, and track over time. Accordingly, before one rushes to an intervention, all treatment considerations need to be ascertained and discussed with the patient and the natural history considered [11]. The latter is especially true in cases of VMT associated with DME, AMD, and RVO where the natural history is unclear and the preferred treatment modality unknown.

In this report, we presented 2 cases of spontaneous resolution of VMT in patients with DME. Indeed, spontaneous resolution is likely to occur in a significant number of these cases given an abnormal vitreal-retinal interface, and we recommend that observation be considered when VMT is associated with relatively good visual acuity and stable DME. Additionally, we postulate that treatments like intravitreal anti-vascular endothelial growth factor may help with resolution of VMT in a fashion similar to sham injections of intravitreal saline in the ocriplasmin clinical trials, and consequently, would support a period of observation as a reasonable course of action. We look forward to studies in similar patients of VMT associated with macular disease as treatment paradigms continue to evolve.

\section{Acknowledgment}

D.R.P.A. was supported by the Retina Foundation of Canada.

\section{Disclosure Statement}

D.R.P.A. has received honoraria from Allergan, Alcon, Genentech, and Novartis. 


\begin{tabular}{l|l}
\hline DOI: $10.1159 / 000360219$ & $\begin{array}{l}\text { C 2014 S. Karger AG, Basel } \\
\text { www.karger.com/cop }\end{array}$ \\
\hline
\end{tabular}

Almeida et al.: Spontaneous Resolution of Vitreomacular Traction in Two Patients with Diabetic Macular Edema

\section{References}

1 Jackson TL, Nicod E, Simpson A, Angelis A, Grimaccia F, Kanavos P: Symptomatic vitreomacular adhesion. Retina 2013;33:1503-1511.

-2 Russell SR, Hageman GS: Optic disc, foveal, and extrafoveal damage due to surgical separation of the vitreous. Arch Ophthalmol 2001;119:1653-1658.

-3 Jackson TL, Nicod E, Angelis A, Grimaccia F, Prevost AT, Simpson AR, Kanavos P: Vitreous attachment in agerelated macular degeneration, diabetic macular edema, and retinal vein occlusion: a systematic review and metaanalysis. Retina 2013;33:1099-1108.

4 Johnson MW: Posterior vitreous detachment: evolution and role in macular disease. Retina 2012;32(suppl 2):S174-S178.

5 Simpson AR, Petrarca R, Jackson TL: Vitreomacular adhesion and neovascular age-related macular degeneration. Surv Ophthalmol 2012;57:498-509.

-6 Sonmez K, Capone A Jr, Trese MT, Williams GA: Vitreomacular traction syndrome: impact of anatomical configuration on anatomical and visual outcomes. Retina 2008;28:1207-1214.

7 Stalmans P, Delaey C, de Smet MD, van Dijkman E, Pakola S: Intravitreal injection of microplasmin for treatment of vitreomacular adhesion: results of a prospective, randomized, sham-controlled phase II trial (the MIVI-IIT trial). Retina 2010;30:1122-1127.

8 Benz MS, Packo KH, Gonzalez V, Pakola S, Bezner D, Haller JA, Schwartz SD: A placebo-controlled trial of microplasmin intravitreous injection to facilitate posterior vitreous detachment before vitrectomy. Ophthalmology 2010;117:791-797.

-9 de Smet MD, Gandorfer A, Stalmans P, Veckeneer M, Feron E, Pakola S, Kampik A: Microplasmin intravitreal administration in patients with vitreomacular traction scheduled for vitrectomy: the MIVI I trial. Ophthalmology 2009;116:1349-1355.

10 Stalmans P, Benz MS, Gandorfer A, Kampik A, Girach A, Pakola S, Haller JA; MIVI-TRUST Study Group: Enzymatic vitreolysis with ocriplasmin for vitreomacular traction and macular holes. N Engl J Med 2012;367:606-615.

11 Odrobina D, Michalewska Z, Michalewski J, Dziegielewski K, Nawrocki J: Long-term evaluation of vitreomacular traction disorder in spectral-domain optical coherence tomography. Retina 2011;31:324331. 
Case Reports in

Ophthalmology

\begin{tabular}{l|l}
\hline Case Rep Ophthalmol 2014;5:66-71 & \\
\hline DOI: 10.1159/000360219 & $\begin{array}{l}\text { ○ 2014 S. Karger AG, Basel } \\
\text { www.karger.com/cop }\end{array}$ \\
\hline
\end{tabular}

Almeida et al.: Spontaneous Resolution of Vitreomacular Traction in Two Patients with Diabetic Macular Edema

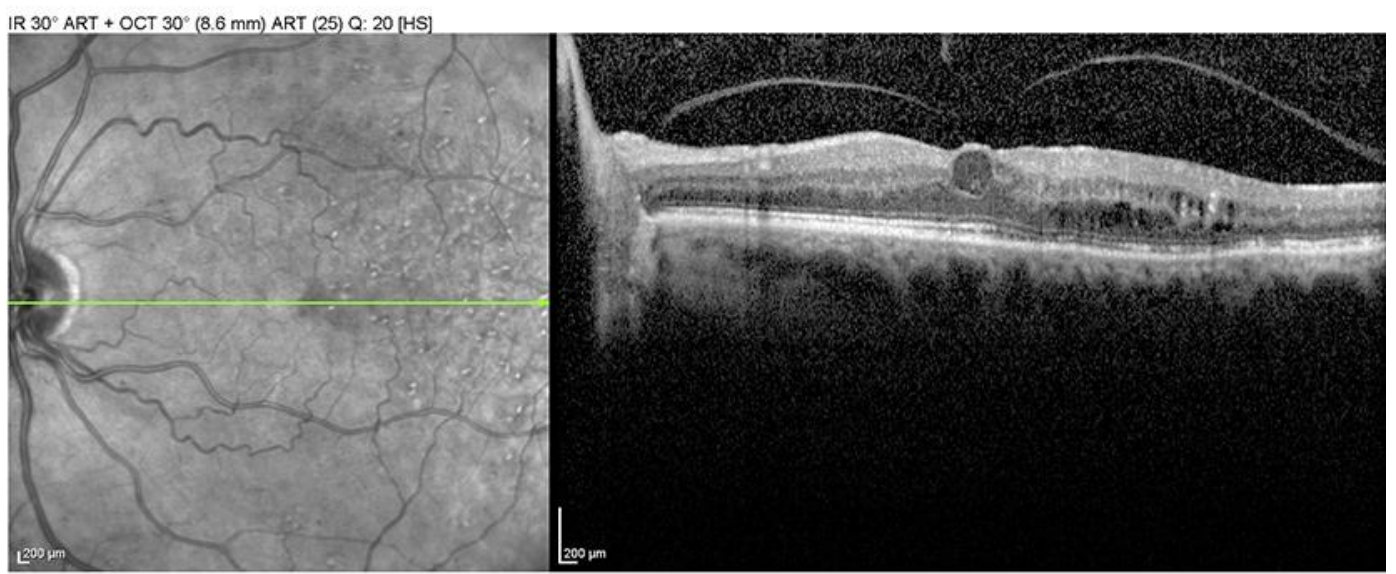

b

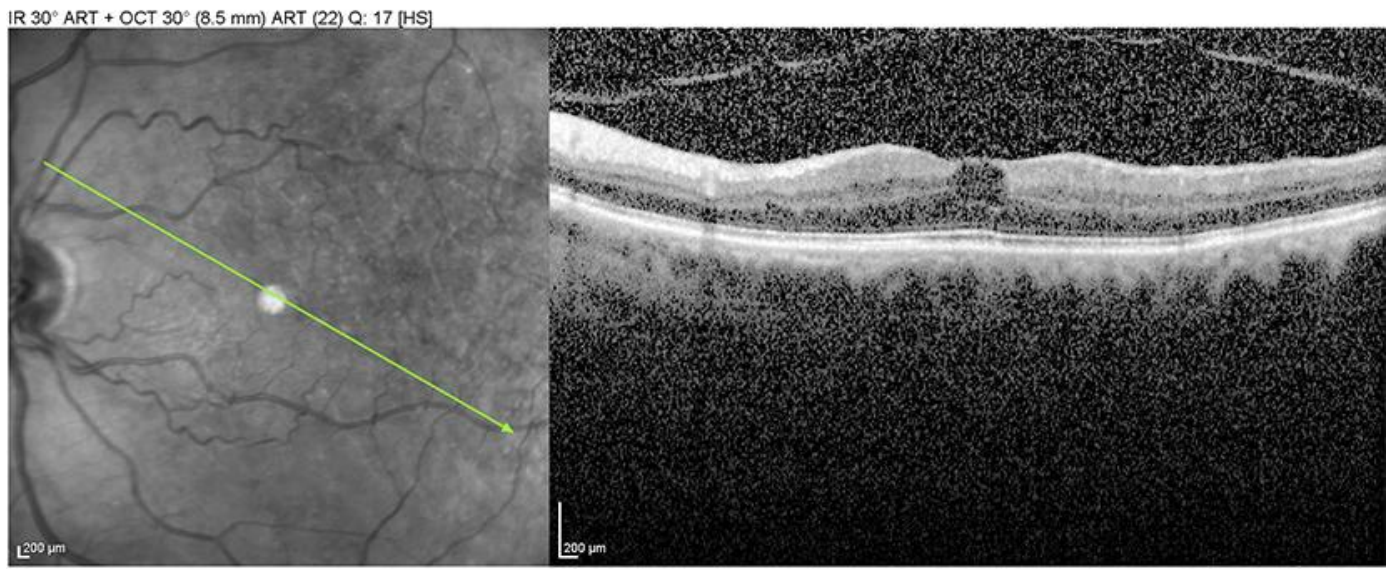

Fig. 1. Spectral domain OCT image of VMT associated with DME. a There is a cystic edema of the fovea and a lamellar edema of the macula temporal to the fovea (CMT $344 \mu \mathrm{m}$ ). There is VMT caused by abnormal vitreomacular adhesion with distortion of the fovea. b After 6 months of observation, the VMT has spontaneously resolved with an improved foveal contour. There is persistent DME (CMT $325 \mu \mathrm{m}$ ). 
Case Reports in

Ophthalmology

\begin{tabular}{l|l}
\hline Case Rep Ophthalmol 2014;5:66-71 \\
\hline DOI: 10.1159/000360219 & $\begin{array}{l}\text { ○ 2014 S. Karger AG, Basel } \\
\text { www.karger.com/cop }\end{array}$ \\
\hline
\end{tabular}

Almeida et al:: Spontaneous Resolution of Vitreomacular Traction in Two Patients with Diabetic Macular Edema

a
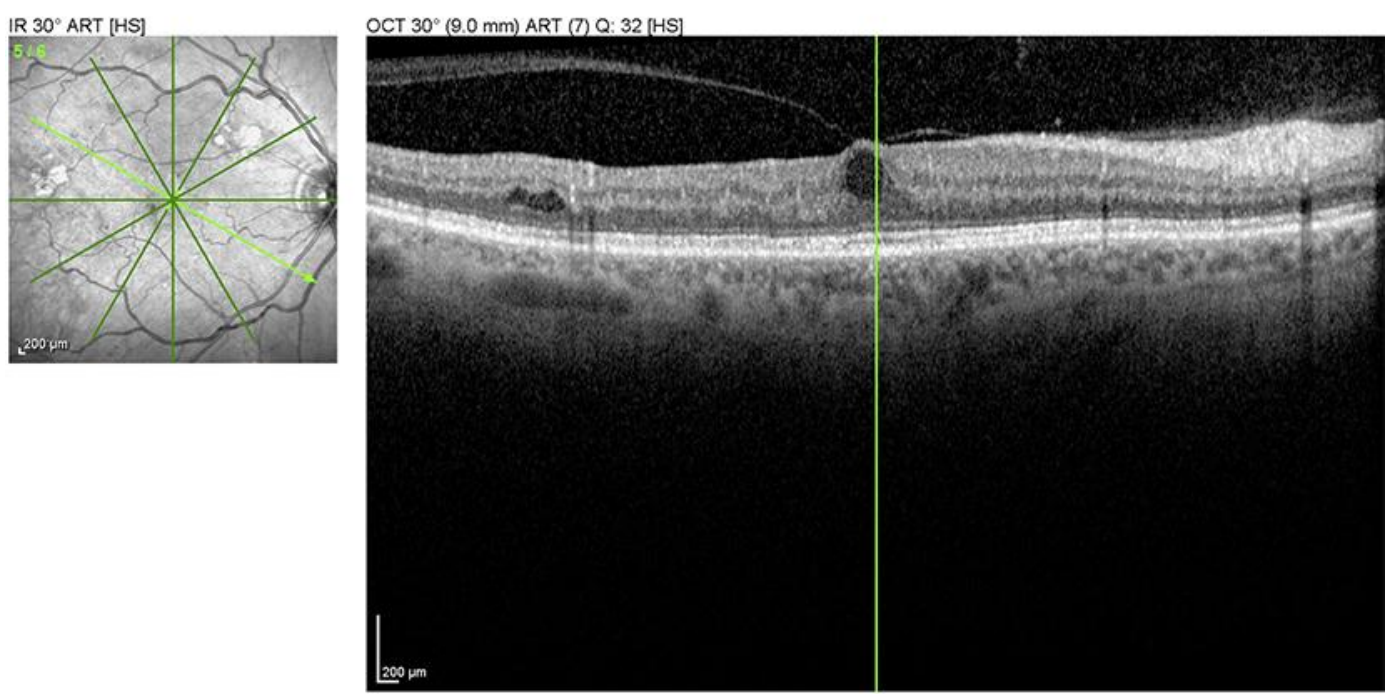

b

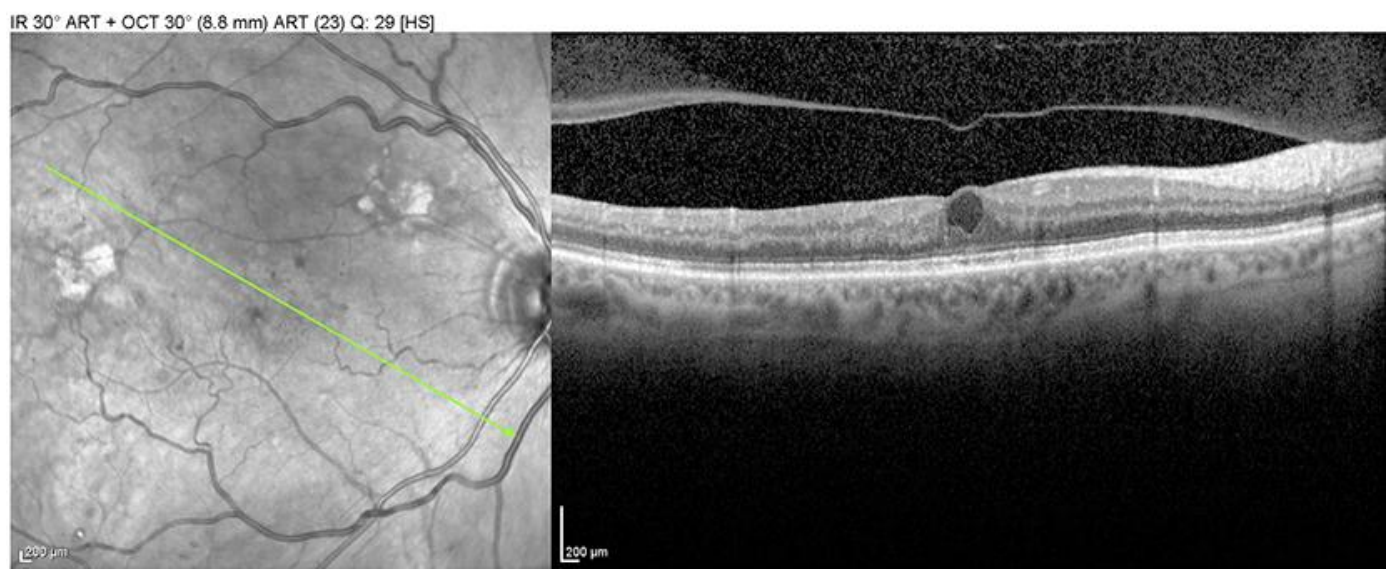

Fig. 2. Spectral domain OCT image of VMT associated with mild DME. a There is a cystic edema of the fovea and a small area of lamellar edema temporal to the fovea (CMT $314 \mu \mathrm{m}$ ). There is VMT at the fovea. $\mathbf{b}$ After 6 months of observation, the VMT has spontaneously resolved with an improved foveal contour. DME is unchanged with a CMT of $314 \mu \mathrm{m}$. 